\title{
Adaptive Decentralized MAC for Event-Triggered Networked Control Systems
}

\author{
Mikhail Vilgelm \\ Chair of Communication \\ Networks \\ Technical University of Munich \\ Munich, Germany \\ mikhail.vilgelm@tum.de
}

\author{
Mohammad H. Mamduhi \\ Chair of Information-oriented \\ Control \\ Technical University of Munich \\ Munich, Germany \\ mh.mamduhi@tum.de \\ Sandra Hirche \\ Chair of Information-oriented \\ Control \\ Technical University of Munich \\ Munich, Germany \\ hirche@tum.de
}

\author{
Wolfgang Kellerer \\ Chair of Communication \\ Networks \\ Technical University of Munich \\ Munich, Germany \\ wolfgang.kellerer@tum.de
}

\begin{abstract}
Control over shared communication networks is a key challenge in design and analysis of cyber-physical systems. The quality of control in such systems might be degraded due to the congestion while accessing the scarce communication resources. In this paper, we consider a multiple-loop networked control system (NCS), where all control loops share a communication network. Medium Access Control (MAC) is performed in contention-based fashion using a multi-channel slotted ALOHA protocol, where each control loop decides locally whether to attempt a transmission based on some error thresholds. We further introduce a local event-based resource-aware scheduling design with an adaptive choice of the error thresholds for a transmission. This leads to a hybrid channel access mechanism where the control loops are deterministically categorized into two sets of eligible and ineligible sub-systems for transmission in an event-based fashion, before a random process to select the available channels. In addition, employing the introduced policy, we show the stability of the resulting NCS in terms of Lyapunov stability in probability. We illustrate numerically the efficiency of our proposed approach in terms of reducing the average networked-induced error variance, and show the superiority of the adaptive event-based scheduler compared to the scheduling design with non-adaptive thresholds.
\end{abstract}

\section{Keywords}

Networked Control Systems; Medium Access Control; Slotted ALOHA; Scheduler Design;

Permission to make digital or hard copies of all or part of this work for personal or classroom use is granted without fee provided that copies are not made or distributed for profit or commercial advantage and that copies bear this notice and the full citation on the first page. Copyrights for components of this work owned by others than ACM must be honored. Abstracting with credit is permitted. To copy otherwise, or republish, to post on servers or to redistribute to lists, requires prior specific permission and/or a fee. Request permissions from permissions@acm.org.

HSCC'16, April 12-14, 2016, Vienna, Austria

(c) 2016 ACM. ISBN 978-1-4503-3955-1/16/04 . \$ $\$ 15.00$

DOI: http://dx.doi.org/10.1145/2883817.2883829

\section{INTRODUCTION}

Traditional digital control systems are characterized by collocated sensors, actuators and controllers as well as by time-triggered control schemes with periodic sampling. With the advent of new technologies, the parts of control systems are becoming spatially distributed and their interaction is being increasingly supported by shared communication networks, which usually impose energy and capacity limitations [22]. In the context of wireless communication, distributed control systems form a specific subset of applications known as Machine-to-Machine (M2M) communication [31]. Smart grids or industrial automation are common examples of M2M use cases.

Control over shared communication resources imposes various challenges, such as congestion due to bandwidth limitation, collisions, and time delays, that compromise the control performance and can even lead to instability. In order to utilize the limited communication and energy resources efficiently, event-triggered control and scheduling schemes have been proposed recently $[6,10,19,20,27,28]$. These aforecited works suggest that it is usually more beneficial to transmit the sampled data upon the occurrence of certain events rather than at periodic time instants. This is even more so in case of large-scale networked control systems due to the sheer amount of data that needs to be exchanged.

In the event-based paradigm, events are typically triggered by either deterministic $[23,30]$, or stochastic policies $[7$, 18, 24, 26]. Deterministic event-based policies award the channel to the entity with the highest priority. Try-OnceDiscard (TOD) is a basic event-based deterministic protocol that awards the medium access to the system with the largest estimation error and consequently discards the other transmission requests [30]. However, TOD is prone to system noise and can cope with collisions only with a given pre-defined priority order, and hence is not convenient for practical realizations [5]. Therefore, an efficient event-based policy for dealing with collisions is still an open research topic.

Due to the non-deterministic transmission patterns of eventbased control systems, and typically long idle periods be- 
tween consecutive transmissions, it is not possible to reserve radio resources for event-based control applications. Thus, it makes them prone to the notorious problem of existing wireless standards, namely, congestion during the connection establishment phase $[8,9,14]$.

The problem has been extensively studied in the context of Long Term Evolution (LTE) Random Access (RA) procedure, where it is commonly modeled as a multi-channel slotted ALOHA system [29]. Many results exist which propose improvements for the LTE RA procedure $[1,12,16,17]$ for general class of M2M devices, however, significantly less contributions can be found in coupling the control system properties and efficient network resource allocation. In $[2,3]$ authors compare the event-based and periodic control via single channel ALOHA for a network of homogeneous integrator sub-systems. Additionally, Cervin et. al. [4] compare different MAC strategies for event-based NCS, however, their assumption about the collision resolution time is basically diminishing the effect of collisions, which is nonnegligible for most of the scenarios. In [21] the authors investigate an adaptive price-based scheduling mechanism for multiple loop NCSs with shared communication resource. In this approach distributed optimization method and adaptive Markov decision process are employed to develop distributed self-regulating event-triggers which are capable of adapting their transmission request rate in order to fulfill a global resource constraint.

In this paper, we analyze the behavior of the multi-channel slotted ALOHA medium access, considering an event-based networked control system consisting of multiple linear timeinvariant (LTI) control sub-systems as the communication endpoints. First, we describe our local threshold-based scheduler which determines whether a sub-system is eligible for a transmission attempt. Stability of the resulting NCS over the multi-channel slotted ALOHA is then discussed in terms of Lyapunov stability in probability (LSP). We evaluate the performance of the event-based scheduler, and further propose an improvement to it, an adaptive scheduler. In the new scheduler design, network and control systems are coupled via the knowledge of the network state: each local scheduler adapts its threshold based on the available network resources. Numerically, we demonstrate that an adaptive choice of the transmission threshold is beneficiary compared to the non-adaptive static design.

The remainder of this paper is structured as follows. We start by introducing the problem statement and preliminaries in Section 2. Stochastic stability of the resulting NCS design in discussed in Section 3. Section 4 is dedicated to the numerical performance evaluation and divided into two parts: Subsection 4.1 illustrates the performance of the static scheduler, and in Subsection 4.2 we demonstrate the benefits of using an adaptive scheduler.

\section{PROBLEM STATEMENT}

In this paper, we consider an NCS consisting of $N$ physically isolated LTI control sub-systems which are coupled through a shared communication network. A control subsystem $i$ is composed of a linear plant $\mathcal{P}_{i}$ and a controller $\mathcal{C}_{i}$. The feedback loop from the plant to the controller is closed via the shared communication network and the decision of whether to attempt the access to the network is taken by the local scheduler $\mathcal{S}_{i}$. The plant process is subject to system noise and can be described with the following stochastic difference equation:

$$
x_{k+1}^{i}=A_{i} x_{k}^{i}+B_{i} u_{k}^{i}+w_{k}^{i},
$$

where $x_{k}^{i} \in \mathbb{R}^{n_{i}}$ denotes the $i^{\text {th }}$ system state at time-step $k, u_{k}^{i} \in \mathbb{R}^{d_{i}}$ describes the control input at time-step $k$. The constant matrices $A_{i} \in \mathbb{R}^{n_{i} \times n_{i}}, B_{i} \in \mathbb{R}^{n_{i} \times d_{i}}$ describe system and input matrices, respectively. The noise sequence $w_{k}^{i}$ is considered to be an independent and identically distributed (i.i.d) vector distributed according to a zero-mean Gaussian distribution with the covariance matrix $W_{i}$. Independent of the noise variables $w_{k}^{i}$, the initial state $x_{0}^{i}$ can be considered to be a random variable of any arbitrary symmetric distribution with bounded second moment. At each time-step $k$, the binary variable $\delta_{k}^{i} \in\{0,1\}$ represents the decision of the local scheduler $\mathcal{S}_{i}$ for sub-system $i$ as follows:

$$
\delta_{k}^{i}= \begin{cases}1, & x_{k}^{i} \text { sent through the channel } \\ 0, & x_{k}^{i} \text { blocked }\end{cases}
$$

Assume that the communication network has $M$ available transmission channels at each time-step (see Fig. 2). According to the multi-channel slotted ALOHA protocol, each sub-system which is eligible for transmission, selects one of the $M$ transmission channels randomly to send its own data packet. We denote number of available channels $M$ as a network state.

A collision would occur if two or more sub-systems select the same channel at a certain sample time $k$. Consequently, none of those sub-systems transmit at $k$ and have to try for transmission at the next time-step $k+1$. (6) A successful transmission (i.e., the scheduled packet is not collided) is confirmed by the binary variable $\gamma_{k}^{i} \in\{0,1\}$ as follows:

$$
\gamma_{k}^{i}= \begin{cases}1, & x_{k}^{i} \text { successfully received } \\ 0, & x_{k}^{i} \text { collided }\end{cases}
$$

Accordingly, the received signal $z_{k}^{i}$ at the controller side of the sub-system $i$ is given as a function of scheduling variable $\delta_{k}^{i}$ and collision indicator $\gamma_{k}^{i}$ as follows:

$$
z_{k}^{i}= \begin{cases}x_{k}^{i}, & \theta_{k}^{i}=1 \\ \varnothing, & \text { otherwise }\end{cases}
$$

where, $\theta_{k}^{i}=\delta_{k}^{i} \gamma_{k}^{i}$. Each sub-system is assumed to be controlled by a state-feedback controller which is updated at every time-step $k$ by either the true state values $x_{k}^{i}$ (in case sub-system $i$ successfully transmits, i.e. $\delta_{k}^{i}=1$ and $\gamma_{k}^{i}=1$ )

Table 1: Summary of most-used notations

\begin{tabular}{|c|l|}
\hline$x_{k}^{i}$ & system state of a sub-system $i$ at time-step $k$ \\
\hline$e_{k}^{i}$ & error state of a sub-system $i$ at time-step $k$ \\
\hline$w_{k}^{i}$ & system noise of a sub-system $i$ at time-step $k$ \\
\hline$A_{i}$ & system matrix of sub-system $i$ \\
\hline$\delta_{k}^{i}$ & scheduling variable \\
\hline$\theta_{k}^{i}$ & transmission indicator \\
\hline$\|\cdot\|$ & Euclidean norm \\
\hline $\mathrm{E}[\cdot \cdot \cdot]$ & conditional expectation operator \\
\hline$\Lambda_{i}$ & error threshold for sub-system $i$ \\
\hline$\Lambda^{\prime}$ & global error threshold for all sub-systems \\
\hline$N$ & total number of control sub-systems \\
\hline$M$ & network state: number of available channels per slot \\
\hline
\end{tabular}


or by the state estimates $\mathrm{E}\left[x_{k}^{i}\right]$ (in case sub-system $i$ is blocked by the scheduler, i.e. $\delta_{k}^{i}=0$ or a collision occurs, i.e. $\left.\gamma_{k}^{i}=0\right)$. It is assumed that the sensor and controller of the $i^{\text {th }}$ sub-system merely have local knowledge, i.e., of $A_{i}, B_{i}, W_{i}$ and the distribution of $x_{0}^{i}$. Therefore, we assume that the control law $\vartheta^{i}$ is described by measurable and causal mapping of the past observations:

$$
u_{k}^{i}=\vartheta_{k}^{i}\left(Z_{k}^{i}\right)=-L_{i} \mathrm{E}\left[x_{k}^{i} \mid Z_{k}^{i}\right]
$$

where $Z_{k}^{i}=\left\{z_{0}^{i}, \ldots, z_{k}^{i}\right\}$ is the $i^{\text {th }}$ controller observation history, and $L_{i}$ is an arbitrary stabilizing feedback gain. In case a transmission fails, either due to a blocking by the scheduler (i.e. $\delta_{k}^{i}=0$ ) or collision (i.e. $\gamma_{k}^{i}=0$ ), the estimate of system state $x_{k}^{i}$ is computed by a model-based estimator as follows:

$$
\mathrm{E}\left[x_{k}^{i} \mid Z_{k}^{i}\right]=\left(A_{i}-B_{i} L_{i}\right) \mathrm{E}\left[x_{k-1}^{i} \mid Z_{k-1}^{i}\right],
$$

with the initial condition $\mathrm{E}\left[x_{0}^{i} \mid Z_{0}^{i}\right]=0$. The estimate (3) is well-behaved only if a stabilizing gain $L_{i}$ exists to ensure that the closed-loop matrix $\left(A_{i}-B_{i} L_{i}\right)$ is Hurwitz. Accordingly, the network-induced estimation error $e_{k}^{i} \in \mathbb{R}^{n_{i}}$ is defined as the difference between the actual and estimated values of the system state, i.e.

$$
e_{k}^{i}:=x_{k}^{i}-\mathrm{E}\left[x_{k}^{i} \mid Z_{k}^{i}\right]
$$

Having the definition (4) and employing (1)-(3), we can derive the dynamics of the networked-induced error state $e_{k}^{i}$. Assume that a sub-system $i$ successfully transmits at timestep $k$, i.e. $\theta_{k}^{i}=1$. Therefore, $z_{k}^{i}=x_{k}^{i}$ and subsequently $u_{k}^{i}=-L_{i} x_{k}^{i}$. Thus, the error at the next time-step can be calculated as:

$$
\begin{aligned}
e_{k+1}^{i} & =x_{k+1}^{i}-\mathrm{E}\left[x_{k+1}^{i} \mid Z_{k+1}^{i}\right] \\
& =A_{i} x_{k}^{i}-B_{i} L_{i} x_{k}^{i}+w_{k}^{i}-\mathrm{E}\left[A_{i} x_{k}^{i}-B_{i} L_{i} x_{k}^{i}+w_{k}^{i} \mid x_{k}^{i}\right] \\
& =\left(A_{i}-B_{i} L_{i}\right) x_{k}^{i}+w_{k}^{i}-\left(A_{i}-B_{i} L_{i}\right) x_{k}^{i} \\
& =w_{k}^{i} .
\end{aligned}
$$

On the other hand, if the sub-system $i$ does not successfully transmit at time-step $k$, i.e. $\theta_{k}^{i}=0$, then $z_{k}^{i}=\varnothing$ and the controller will be updated by the estimated value of $x_{k}^{i}$, i.e. $u_{k}^{i}=-L_{i} \mathrm{E}\left[x_{k}^{i} \mid Z_{k}^{i}\right]$. In this case, we have

$$
\begin{aligned}
& e_{k+1}^{i}=A_{i} x_{k}^{i}-B_{i} L_{i} \mathrm{E}\left[x_{k}^{i} \mid Z_{k}^{i}\right]+w_{k}^{i} \\
& \quad-\mathrm{E}\left[A_{i} x_{k}^{i}-B_{i} L_{i} \mathrm{E}\left[x_{k}^{i} \mid Z_{k}^{i}\right]+w_{k}^{i} \mid Z_{k}^{i}\right] \\
& \quad=A_{i} x_{k}^{i}-B_{i} L_{i} \mathrm{E}\left[x_{k}^{i} \mid Z_{k}^{i}\right]+w_{k}^{i}-A_{i} \mathrm{E}\left[x_{k}^{i} \mid Z_{k}^{i}\right]+B_{i} L_{i} \mathrm{E}\left[x_{k}^{i} \mid Z_{k}^{i}\right] \\
& =A_{i}\left(x_{k}^{i}-\mathrm{E}\left[x_{k}^{i} \mid Z_{k}^{i}\right]\right)+w_{k}^{i} \\
& =A_{i} e_{k}^{i}+w_{k}^{i} .
\end{aligned}
$$

Rewriting the error dynamics for the general $\theta_{k}^{i}$, we obtain the following form:

$$
e_{k+1}^{i}=\left(1-\theta_{k}^{i}\right) A_{i} e_{k}^{i}+w_{k}^{i} .
$$

The decision whether to attempt a transmission or not is taken by the scheduler $\mathcal{S}_{i}$ described in the next Subsec. 2.1.

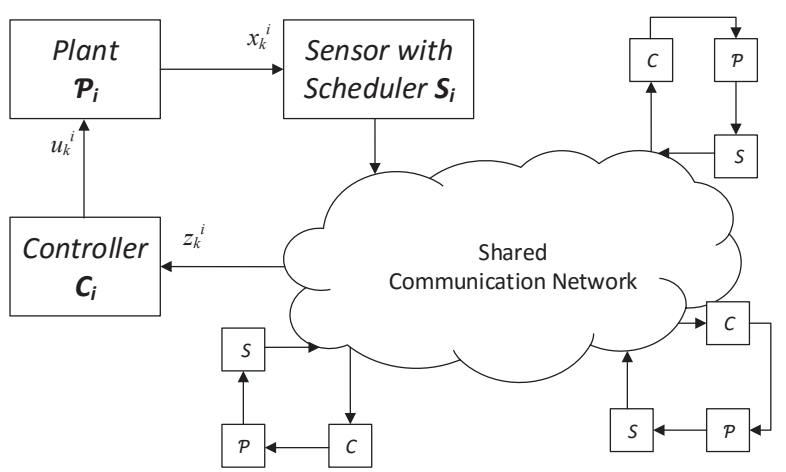

Figure 1: A multi-loop NCS with a shared communication channel and local scheduling mechanism.

\subsection{Local threshold-based scheduler}

The local scheduler situated at each local control loop decides to access the medium at every time-step $k$ only if the following threshold inequality holds:

$$
\left\|e_{k}^{i}\right\|>\Lambda_{i}
$$

where, $\Lambda_{i}$ is the local error threshold for sub-system $i$. Therefore, if (6) is satisfied at some time-step $k$, then the corresponding sub-system is eligible for transmission at the next time-step $k+1$. Otherwise, it is deterministically excluded from the channel access, i.e.

$$
\mathrm{P}\left[\delta_{k+1}^{i}=1 \mid e_{k}^{i}\right]= \begin{cases}0, & \text { if }\left\|e_{k}^{i}\right\| \leq \Lambda_{i} \\ 1, & \text { otherwise. }\end{cases}
$$

Note that the deployed scheduling policy (7) is not explicitly dependent on the whether the transmission has been successful or it has collided, therefore, channel sensing of acknowledgements are not necessary for the policy's realization.

The communication network model is restricted to the Media Access Layer (MAC) and is represented by a multichannel slotted ALOHA protocol [25], see Fig. 2. As the most common practical example, we can refer to LTE-based system and its Random Access Channel [9], while mappings to different single-hop wireless or even bus systems can also be imagined. In every time slot, there are several non-overlapping transmission channels available. We denote the number of available channels as $M$. As we investigate the multi-channel model in this paper, we assume $M \geq 2$.

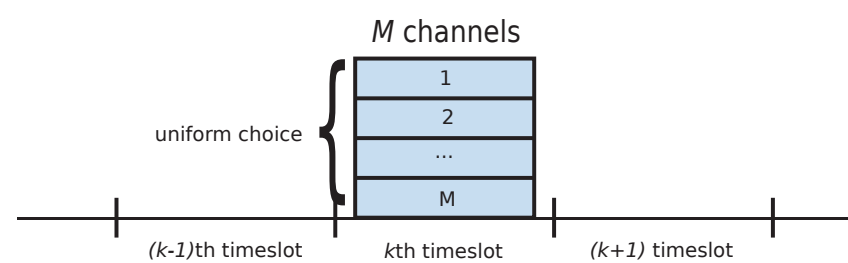

Figure 2: Communication system model: multichannel slotted ALOHA. One time slot is assumed equal to a control period of any sub-system. A channel can represent a frequency, code [29] or time domain transmission opportunity, depending on the communication technology in use. 
The information about the available number of channels is assumed to be known for all sub-systems in the beginning of each time slot.

For the sake of simplicity, we assume that the communication time slots are equal in duration to the control sampling periods, and that all sub-systems' control periods are synchronized. Thus, in every control period we have $M$ available transmission channels, meaning:

$$
\sum_{i=1}^{N} \theta_{k}^{i} \leq M
$$

According to the slotted ALOHA protocol, if a packet is scheduled for transmission, it will be sent through one of $\mathrm{M}$ channels, randomly chosen. Thus, if we denote a set of sub-systems which are eligible for transmission at time-step $k$ as $\mathcal{G}_{k}$, then the probability of successful transmission for a given eligible sub-system in the $k$ timestep is calculated as:

$$
\mathrm{P}\left[\gamma_{k+1}^{i}=1 \mid\left\|e_{k}^{i}\right\|>\Lambda_{i}\right]=\left(\frac{M-1}{M}\right)^{g_{k}},
$$

where $g_{k}$ is the cardinality of the set $\mathcal{G}_{k}$.

The transmission threshold $\Lambda_{i}$ is influencing directly both the error of the sub-system, and the arrival rate of the requests for network access. Since the network is modeled by slotted ALOHA mechanism, too high arrival rate of requests will result in a high collision rate and consequently degrades the performance of the overall networked system, significantly. Following this observation, our hypothesis is that adapting $\Lambda_{i}$ to network state, can be beneficial for the control performance.

\section{STABILITY ANALYSIS}

In this section, we study stability of multiple-loop NCSs with shared multi-channel communication networks subject to the constraint (8), and the introduced threshold-based decentralized scheduling policy (7). We show stochastic stability of the overall networked system by the notion of Lyapunov stability in probability (LSP). Before introducing the notion of LSP, we will describe the overall network state at some time-step $k$ by the aggregation of the system states $x_{k}^{i}$ from all sub-systems $i \in\{1, \ldots, N\}$ and error states $e_{k}^{i}$ from all sub-systems $i \in\{1, \ldots, N\}$, i.e. $\left[x_{k}^{\top}, e_{k}^{\top}\right]^{\top}$, where $x_{k}=\left[x_{k}^{1^{\top}}, \ldots, x_{k}^{N^{\top}}\right]^{\top}$ and $e_{k}=\left[e_{k}^{1^{\top}}, \ldots, e_{k}^{N^{\top}}\right]^{\top}$. From (1)(3), together with the definition of the estimation error $e_{k}^{i}$ in (4), it is straightforward to see that the individual aggregate networked state $\left[x_{k}^{i^{\top}}, e_{k}^{i} \mathrm{~T}\right]^{\top}$ within each sub-system $i$ has triangular dynamics as follows:

$$
\left[\begin{array}{c}
x_{k+1}^{i} \\
e_{k+1}^{i}
\end{array}\right]=\left[\begin{array}{cc}
A_{i}-B_{i} L_{i} & \left(1-\theta_{k}^{i}\right) B_{i} L_{i} \\
0 & \left(1-\theta_{k}^{i}\right) A_{i}
\end{array}\right]\left[\begin{array}{c}
x_{k}^{i} \\
e_{k}^{i}
\end{array}\right]+\left[\begin{array}{c}
w_{k}^{i} \\
w_{k}^{i}
\end{array}\right]
$$

This implies that the evolution of the error state $e_{k}^{i}$ is in fact independent of the system state $x_{k}^{i}$. We employ an emulation-based control design to stabilize the control subsystems in case their corresponding loops are closed, i.e. the controllers are updated with their own true state values. This incurs that, assuming each pair $\left(A_{i}, B_{i}\right)$ is stabilizable, there exists stabilizing feedback gain $L_{i}$ such that the closedloop matrix $\left(A_{i}-B_{i} L_{i}\right)$ is Hurwitz, and consequently the system state $x_{k}^{i}$ is asymptotically stable. It should however be noted that existence of stabilizing control laws $u_{k}^{i}$ 's does not guarantee the stability of overall networked system with the introduced networked state $\left.\left[x_{k}^{i^{\top}}, e_{k}^{i}\right]^{\top}\right]^{\top}$, since the evolution of the error state is independent of the control laws. This statement is clear from (10), which illustrates that if a sub-system does not transmit at a certain time-step, stabilizing gain $L_{i}$ guarantees the stability only if error state $e_{k}^{i}$ is stable. Now we are ready to introduce the concept of stability, i.e. LSP, considered in this paper.

Definition 1. (Lyapunov Stability in Probability (LSP), [13]) A linear system with state vector $x_{k}$ possesses LSP if given $\varepsilon, \varepsilon^{\prime}>0$, exists $\rho\left(\varepsilon, \varepsilon^{\prime}\right)>0$ such that $\left|x_{0}\right|<\rho$ implies

$$
\lim _{k \rightarrow \infty} \sup \mathrm{P}\left[x_{k}^{T} x_{k} \geq \varepsilon^{\prime}\right] \leq \varepsilon \text {. }
$$

The following lemma shows the LSP is achievable by solely considering the error state $e_{k}$ in our NCS of interest.

Lemma 1. For an NCS described by (1)-(5), the condition in (11) is equivalent to

$$
\lim _{k \rightarrow \infty} \sup \mathrm{P}\left[e_{k}^{T} e_{k} \geq \xi^{\prime}\right] \leq \xi
$$

where $\xi^{\prime}>0$ and the constant $\xi$ fulfills $0 \leq \xi \leq \varepsilon$.

PROOF. As already stated, the system state $x_{k}^{i}$ for each control loop $i$ evolves as

$$
x_{k+1}^{i}=\left(A_{i}-B_{i} L_{i}\right) x_{k}^{i}+\left(1-\theta_{k}^{i}\right) B_{i} L_{i} e_{k}^{i}+w_{k}^{i} .
$$

As already discussed, the evolution of the error $e_{k}^{i}$ is independent of the system state $x_{k}^{i}$ within each individual control loop. Furthermore, by assuming the emulative control law (2), the closed-loop matrix $\left(A_{i}-B_{i} L_{i}\right)$ is ensured to be Hurwitz. Together with the assumption that $x_{0}^{i}$ has a symmetric bounded variance distribution, it follows that the system state $x_{k}^{i}$ is converging with any stabilizing feedback gain $L_{i}$. In addition, the disturbance process $w_{k}^{i}$ is i.i.d. according to $\mathcal{N}(0, I)$, and is bounded in probability. Thus, showing $\lim _{k \rightarrow \infty} \sup \mathrm{P}\left[e_{k}^{i^{\top}} e_{k}^{i} \geq \xi_{i}^{\prime}\right] \leq \xi_{i}$ ensures existence of constants $\varepsilon_{i}$ and $\varepsilon_{i}^{\prime}>0$ such that $\lim _{k \rightarrow \infty} \sup \mathrm{P}\left[x_{k}^{i^{\top}} x_{k}^{i} \geq \varepsilon_{i}^{\prime}\right] \leq \varepsilon_{i}$, where $\xi_{i} \leq \varepsilon_{i}$. As individual loops operate independently, we take the aggregate NCS state $\left(x_{k}, e_{k}\right)$. Then, the existence of $\xi$ and $\xi^{\prime}>0$ such that $\lim _{k \rightarrow \infty} \sup \mathrm{P}\left[e_{k}^{\top} e_{k} \geq \xi^{\prime}\right] \leq \xi$, implies existence of $\varepsilon$ and $\varepsilon^{\prime}>0$ such that $\lim _{k \rightarrow \infty} \sup \mathrm{P}\left[x_{k}^{\top} x_{k} \geq \varepsilon^{\prime}\right] \leq \varepsilon$ for $\xi \leq \varepsilon$, and the proof readily follows.

This lemma enables us to study stability of the overall networked system only by looking at the error state $e_{k}$, considering that stabilizing feedback gains $L_{i}$ are designed.

As expected values are more straightforward in pursuing further analysis than probabilities, we employ the following inequality for $\xi^{\prime}>0$ as

$$
\mathrm{P}\left[e_{k}^{\top} e_{k} \geq \xi^{\prime}\right] \leq \frac{\mathrm{E}\left[e_{k}^{\top} e_{k}\right]}{\xi^{\prime}} .
$$

The above expression is derived using Markov's inequality. This confirms that showing the error is uniformly bounded in expectation ensures finding appropriate $\xi$ and $\xi^{\prime}>0$ such that $(12)$ is satisfied for arbitrary $\rho\left(\xi^{\prime}, \xi\right)$. Therefore, we focus on deriving an upper bound for the expectation of quadratic error norm, i.e.

$$
\mathrm{E}\left[e_{k}^{\top} e_{k}\right]=\sum_{i=1}^{N} \mathrm{E}\left[e_{k}^{i^{\top}} e_{k}^{i}\right]=\sum_{i=1}^{N} \mathrm{E}\left[\left\|e_{k}^{i}\right\|^{2}\right]
$$


This modifies the condition (12) as follows:

$$
\lim _{k \rightarrow \infty} \sup \mathrm{P}\left[e_{k}^{\top} e_{k} \geq \bar{\xi}^{\prime}\right] \leq \bar{\xi}
$$

Due to the nature of the multi-channel communication network with capacity constraint (8), and threshold-based scheduler policy (7), the boundedness of (15) cannot always be shown over one time-step transition, i.e. $k \rightarrow k+1$. This observation is discussed in the following illustrative example: Illustrative example Consider an NCS consisting of three identical scalar unstable sub-systems with systems matrices $A_{1}=A_{2}=A_{3}=A>1$, competing for two available transmission channels at each time slot over a shared multi-channel communication network. For simplicity, assume $\Lambda_{1}=\Lambda_{2}=\Lambda_{3}=\bar{\Lambda}$, and $e_{k}^{1}=e_{k}^{2}=e_{k}^{3}=\bar{e}_{k}$. In addition, consider that the condition (6) is fulfilled, i.e. all three sub-systems are eligible for channel access at timestep $k+1$. Each sub-system selects each of the two available transmission channels by probability of $\frac{1}{2}$. Two scenarios are viable: 1) one successful transmission occurs from one of the sub-systems, and the other two will inevitably collide. It is straightforward to calculate that this scenario happens with the probability of $\frac{3}{4} ; 2$ ) all three sub-systems choose the same transmission channel and consequently all three will collide, which means no successful transmission is occurred, where this scenario occurs with probability of $\frac{1}{4}$. As the sub-systems are identical, and for the sake of illustrative purposes, assume a realization for the first scenario that e.g. sub-system 1 transmits and sub-systems 2 and 3 are collided. Employing (5), we calculate the error expectation in (15) for one step transition, as follows:

$$
\begin{aligned}
& \sum_{i=1}^{3} \mathrm{E}\left[\left\|e_{k+1}^{i}\right\|^{2} \mid e_{k}\right]=\sum_{i=1}^{3} \mathrm{E}\left[\left\|\left(1-\theta_{k}^{i}\right) A e_{k}^{i}+w_{k}^{i}\right\|^{2}\right] \\
& =\frac{1}{4} \sum_{i=1}^{3} \mathrm{E}\left[\left\|A \bar{e}_{k}+w_{k}^{i} \mid \bar{e}_{k}\right\|^{2}\right] \\
& +\frac{3}{4}\left(\mathrm{E}\left[\left\|A \bar{e}_{k}+w_{k}^{2} \mid \bar{e}_{k}\right\|^{2}\right]+\mathrm{E}\left[\left\|A \bar{e}_{k}+w_{k}^{3} \mid \bar{e}_{k}\right\|^{2}\right]+\mathrm{E}\left[\left\|w_{k}^{1}\right\|^{2}\right]\right) \\
& =\frac{1}{4} \sum_{i=1}^{3}\left\|A \bar{e}_{k}\right\|^{2}+\mathrm{E}\left[\left\|w_{k}^{i}\right\|^{2}\right] \\
& +\frac{3}{4}\left(2\left\|A \bar{e}_{k}\right\|^{2}+\sum_{i=1}^{3} \mathrm{E}\left[\left\|w_{k}^{i}\right\|^{2}\right]\right) \\
& =\frac{1}{4}\left(3\left\|A \bar{e}_{k}\right\|^{2}+3\right)+\frac{3}{4}\left(2\left\|A \bar{e}_{k}\right\|^{2}+3\right) \\
& =3+2.25\left\|A \bar{e}_{k}\right\|^{2}
\end{aligned}
$$

which is not uniformly bounded for arbitrary $\bar{e}_{k}$ and system matrix $A$. Intuitively, between two consecutive transmissions of each sub-system, they operate in open loop. Hence, in general, the respective local errors are expected to grow. Thus to obtain boundedness of error state, we need to look at an interval of time-steps rather than only one transition step such that, given the channel capacity constraint (8), all sub-systems have non-zero chances of transmission. Therefore, one can infer that an interval of length $\left|\frac{N}{M-1}\right|$ provides enough transmission possibilities for an NCS of $N$ subsystems and $M$ available transmission channels per timestep. It should be reminded that the linearity of our subsystems guarantees the boundedness over any finite longer horizons.

For stability analysis, we assume the worst case scenario by considering the minimum number of available transmission channels, i.e. only two transmission channels at each time-step. This yields that the minimum length of the interval over which LSP is investigated equals $N$.

THEOREM 1. Consider an NCS with $N$ heterogeneous LTI control sub-systems, with the plants given by (1), sharing a multi-channel communication network with two available transmission channels per time-step. Given the control law (2) and threshold policy (7), the NCS of interest is Lyapunov stable in probability if the MAC employs slotted ALOHA protocol.

\section{Proof. See Appendix A.}

REMARK 1. The notion of stability considered in this paper, i.e., LSP, determines the probability that the overall NCS state remain bounded. This probability is not one due to the fact that there exists a non-zero probability, though might be very close-to-zero, such that at all time-steps the NCS is operating all the transmissions fail due to successive collisions. This is the structural property of the decentralized MAC we are considering in this paper and in case such a scenario occurs, it means all control loops, either stable or unstable, operate in open-loop which consequently lead to instability of the overall NCS due to the presence of unstable plants.

\section{PERFORMANCE EVALUATION}

In this section, we evaluate the performance of a thresholdbased scheduler over multi-channel slotted aloha. Both communication and control-related aspects are investigated.

For the simulations, we consider an evaluation setup as follows. An NCS in consideration is composed of two heterogeneous classes of scalar control loops: class one including multiple homogeneous stable plants with the system matrix $A_{1}=0.75$ and class two consisting of unstable plants with $A_{2}=1.25$. The plants within each group are homogeneous, and all sub-systems are influenced by the i.i.d. noise processes randomly chosen from the standard normal distribution, i.e. $w_{k}^{i} \sim \mathcal{N}(0,1)$ for all time-steps $k$. The input matrices for both groups are $B_{1}=B_{2}=1$. For the plants' stabilization, deadbeat control law $L_{i}=A_{i}$ is employed. We consider the total amount of sub-systems to be $N$, while each group of control loops has $N / 2$ sub-systems. The number of transmission channels in each slot, unless stated otherwise, is considered to be $M=10$. It is worth mentioning that not only stability or instability of a plant determines the urge of a transmission, but also system noise influences the threshold-based policy. Therefore, it is not guaranteed that if a plant is stable, then it is asymptotically stable even if no transmission is associated with that sub-system. Due to presence of noise, a sub-system with stable plant might become in more urgent situation for transmission than a sub-system with unstable plant.

For a control performance evaluation, we study the average error variance among $N$ sub-systems:

$$
\Sigma=\frac{1}{N} \sum_{i=1}^{N} \operatorname{var}\left[e_{k}^{i}\right]
$$

From the communication point of view, we use two metrics. First one is average channel utilization, commonly known as throughput $T$, defined as:

$$
T=\frac{\mathrm{E}\left[n_{p}^{s}\right]}{M}
$$




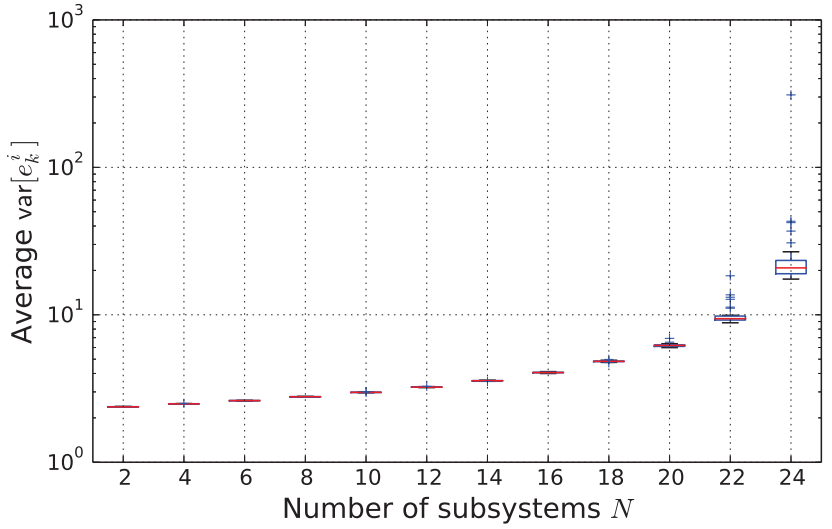

Figure 3: Average error variance $\operatorname{var}\left[e_{k}^{i}\right]$ vs. number of sub-systems $N$ (30 runs): $M=10, \Lambda^{\prime}=2$.

where $\mathrm{E}\left[n_{p}^{s}\right]$ is expected number of successful transmissions per slot. Ratio of collided packets is used as the second performance metric. It is defined as:

$$
r_{\text {coll }}=\frac{\mathrm{E}\left[n_{p}^{c}\right]}{\mathrm{E}\left[n_{p}^{c}\right]+\mathrm{E}\left[n_{p}^{s}\right]},
$$

where $\mathrm{E}\left[n_{p}^{c}\right]$ is expected number of collided transmissions per slot.

The transmission threshold $\Lambda_{i}$ is considered homogeneous for all $N$ sub-systems throughout the simulation:

$$
\Lambda_{i}=\Lambda_{j}, \quad \forall i, j \in N .
$$

To simplify the notations, we denote it by $\Lambda^{\prime}$.

\subsection{Static Threshold Scheduler}

For the first setup we consider a scheduler, where the transmission threshold is chosen arbitrary and is independent of the number of transmission channels $M$.

Fig. 3 demonstrates the evolution of the average error variance with the increasing number of sub-systems. We observe a non-linear growth of the error variance, and, on the same time, higher variation of the resulting variance over multiple runs. The growth of the error variance can be explained by looking at Fig. 5: with the increasing number of subsystems we see an increase in collision rate. Since for the unstable systems, the error accumulates exponentially with every collision, linear increase in collisions results in a nonlinear increase in the variance of the error.

In Fig. 5, we observe that the shape of the plot for throughput corresponds to the commonly known dependency for multi- and single-channel slotted aloha with Poisson distribution arrival rate [29]. The highest value $T \approx 1 / e \approx 0.368$ is achieved at $N=26$.

Fig. 4 shows how the error variance depends on the transmission threshold $\Lambda^{\prime}$. As we observe, and it is inline with the hypothesis we have stated in the Section 2, the dependency is a convex function. With the values of $\Lambda^{\prime}$ close to 0 , the transmission is attempted every time, thus, causing many collisions and shifting the throughput $T$ operating region as in 5 to the right. The collisions, in turn, further increase the $\left\|e_{k}^{i}\right\|$ for all unstable systems with $A_{i}>1$, thus, further increasing the amount of access attempts. As expected, the error variance among all sub-systems grows. If, however,

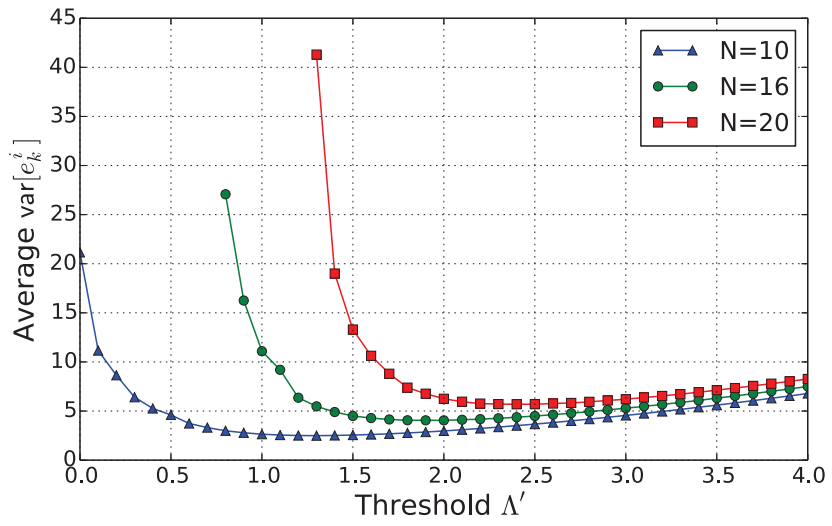

Figure 4: Average error variance $\operatorname{var}\left[e_{k}^{i}\right]$ vs. $\Lambda^{\prime}$. Parameters: $M=10$.

Table 2: Optimal $\Lambda^{\prime}=f(N, M)$.

\begin{tabular}{|l|c|c|c|c|c|c|c|}
\hline & \multicolumn{7}{|c|}{$\mathbf{N}$} \\
\hline & 4 & 6 & 8 & 10 & 12 & 14 & 16 \\
\hline$M=5$ & 1.0 & 1.5 & 2.0 & 2.4 & 3.5 & 5.2 & 8.1 \\
\hline$M=10$ & 0.6 & 0.8 & 1.0 & 1.2 & 1.4 & 1.6 & 1.8 \\
\hline
\end{tabular}

the $\Lambda^{\prime}$ is chosen too high, the increase in the error variance is caused by the underutilized communication medium (throughput $T$ low). Thus, it is observed that there exists an optimal value for $\Lambda^{\prime}$ in a given NCS scenario defined by $N, M$.

\subsection{Scheduler with Threshold Adaptation}

Following the observation about the existence of an optimal $\Lambda^{\prime}$, we propose an improvement to the threshold design defined in (21). Namely, we use a knowledge about the network state $M$ and the number of present sub-systems $N$, in order to choose the $\Lambda^{\prime}$ for the optimal performance:

$$
\Lambda^{\prime}=f(M),
$$

where higher number of channels results in a higher $\Lambda^{\prime}$.

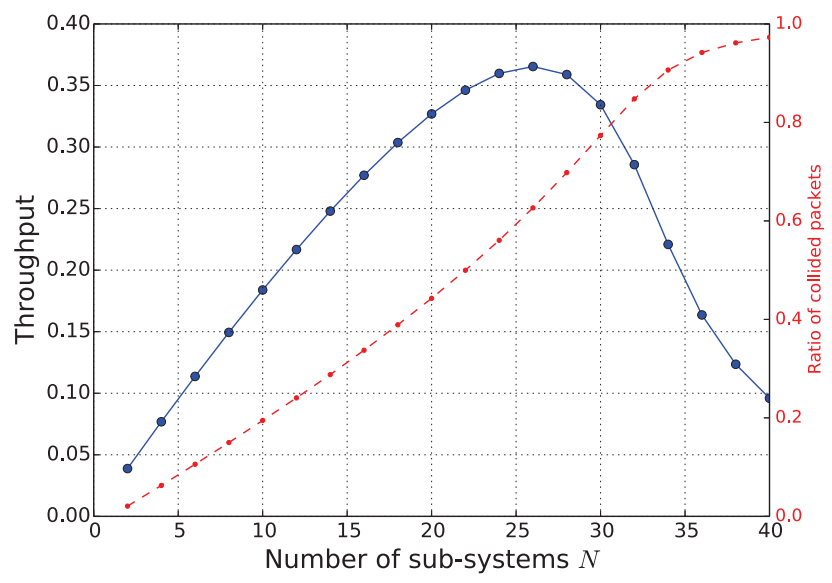

Figure 5: Average throughput and collision rate vs. number of sub-systems $N$. Parameters: $M=10$. 


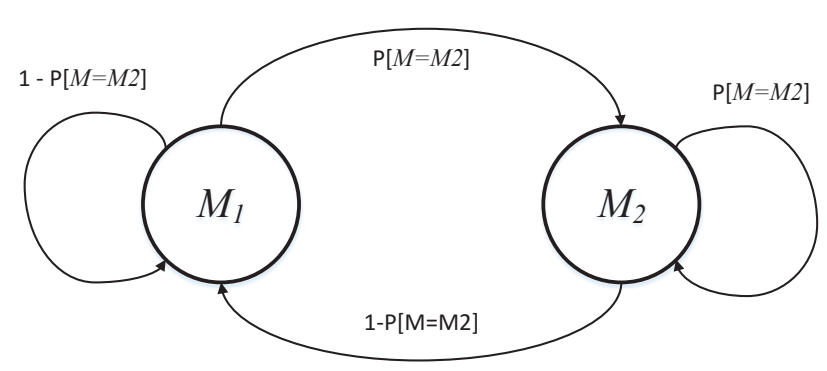

Figure 6: Model of number of channels $M$ variations.

Numerically obtained values for $\Lambda^{\prime}$ for $M$ and $N$ choices we use for evaluation are summarized in Table 2.

The benefits of this approach can be seen for the case of the varying number of available channels $M$. For simplicity, we model the number of channels as a random variable with two possible values $M \in\left\{M_{1}, M_{2}\right\}, M_{1}<M_{2}$, with:

$$
\mathrm{P}[M=M 1]=1-\mathrm{P}[M=M 2] .
$$

The model is depicted in Fig. 6. These two states can represent presence or absence of a background traffic with reserved channels, for example, as described in [11,15]. Although we consider only two states for $M$, it has to be noted that the proposed scheduler design is extendable for a more general case of multiple states. In the evaluation scenario $M_{1}=5$ and $M_{2}=10$, and $\mathrm{P}[M=M 1]=0.5$ are used.

For comparison, we consider two choices of $\Lambda^{\prime}$ for static scheduler: (A) first, where $\Lambda^{\prime}$ is statically set to minimize the error variance for $M=M_{1}$, and (B) second to minimize the error variance for $M=M_{2}$ for a given number of sub-systems in the simulation $N$. The comparison results are presented in Fig. 7. It is observed, that the error variance with the adaptive scheduler is always lower or equal than for non-adaptive. It is further observed, that the first static scheduler (A), optimizing the threshold for the lower number of channels $M_{2}$ is performing noticeably better than the scheduler (B), optimizing the threshold for the higher number of channels $M_{1}$. The effect is supported by the ob-

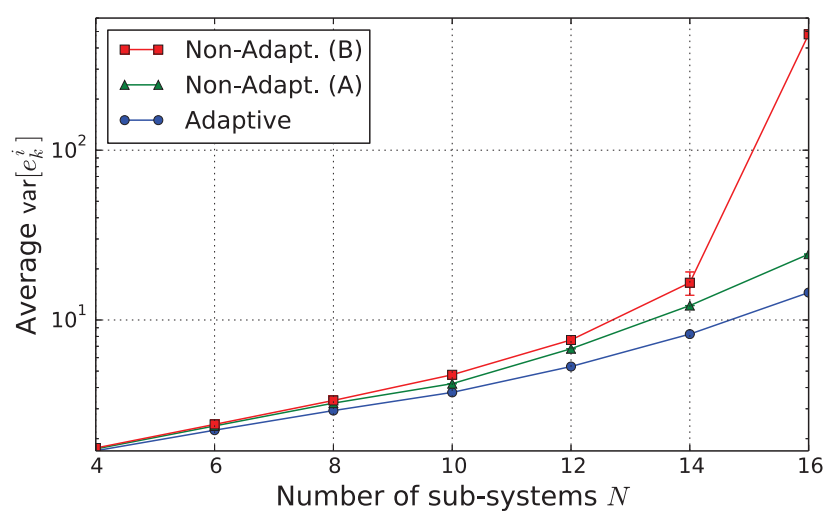

Figure 7: Average error variance vs. number of sub-systems $N$ for three cases: Adaptive $\Lambda^{\prime}$, Non-Adaptive ( $\Lambda^{\prime}$ optimal for $M 1$ channels), NonAdaptive ( $\Lambda^{\prime}$ optimal for $M 2$ channels). Parameters: $M_{1}=5, M_{2}=10, \mathrm{P}\left[M=M_{2}\right]=0.5$.

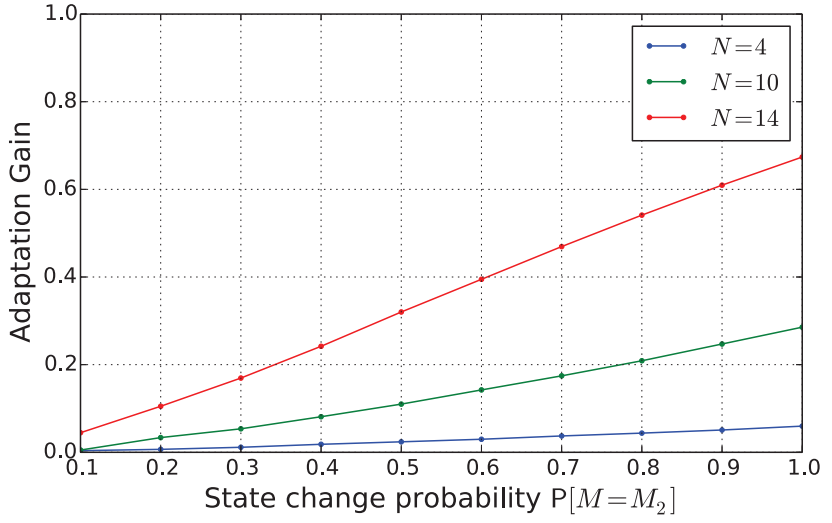

Figure 8: Adaptation gain $G_{a d a p}$ vs. Probability of the "good" channel $\mathrm{P}[M=M 2]$ for $N \in\{4,10,14\}$. Parameters: $M_{1}=5, M_{2}=10$.

servations from Fig. 4 that the slope on the left from the optimal point is much higher than on the right from it, thus, over-utilization is more harmful for the error variance than underutilization.

To evaluate how the probability of a network state change $\mathrm{P}\left[M=M_{2}\right]$ influences the performance gain from the adaptive scheduler, we use the reduction of the average error variance as a metric for adaptation gain:

$$
G_{\text {adap }}=\frac{\Sigma_{n a}-\Sigma_{a}}{\Sigma_{n a}},
$$

where $\Sigma_{n a}$ and $\Sigma_{a}$ represent the average error variances for static (non-adaptive) and adaptive schedulers, respectively.

The resulting dependency is depicted in Fig. 8. The parameter $\mathrm{P}[M=M 2]$ is in this case a measure of how frequently the network state is changing. For $\mathrm{P}[M=M 2]=$ 0.1 almost no changes are there, hence, both schedulers are close to optimal. On the other hand, for $\mathrm{P}[M=M 2]=1$, although also no changes are present, the default state of the channel is $M=M 2$, thus, the static scheduler is not optimal in any time-slot. For the network state changing every second time, the adaptive scheduler is able to reduce the error variance by up to $30 \%$.

\section{CONCLUSIONS}

In this paper, we propose a decentralized threshold-based scheduling policy for an NCS composed of multiple heterogeneous LTI control loops operating via a multi-channel shared communication medium. The Medium Access Control (MAC) is assumed to be performed in contentious fashion using a multi-channel slotted ALOHA protocol. The analysis in the paper proves stochastic stability of such NCSs.

After demonstrating that there exists an global threshold value minimizing the average error variance, we further introduce a local resource-aware scheduler design with an adaptive choice of the error threshold based on knowledge of the network state, and numerically demonstrate that by deploying it instead of the static threshold choice, we can significantly increase the control performance. Future work aims at finding the exact relation or a close approximation of the relation between the network state and the optimal transmission threshold. 


\section{ACKNOWLEDGMENTS}

This work has been in parts supported by TUM Institute for Advanced Study.

\section{REFERENCES}

[1] 3GPP. Technical Report 37.868: Study on RAN Improvements for Machine-type Communications (Release 11). Technical report, 3GPP Technical Specification Group Radio Access Network, 2011.

[2] R. Blind and F. Allgöwer. Analysis of Networked Event-Based Control with a Shared Communication Medium: Part I-Pure ALOHA. Jeb, 1:2, 2011.

[3] R. Blind and F. Allgöwer. Analysis of networked event-based control with a shared communication medium: Part II-Slotted ALOHA. In IFAC World Congress, pages 8830-8835, 2011.

[4] A. Cervin and T. Henningsson. Scheduling of event-triggered controllers on a shared network. In Decision and Control, 2008. CDC 2008. 47th IEEE Conference on, pages 3601-3606, Dec 2008.

[5] D. Christmann, R. Gotzhein, S. Siegmund, and F. Wirth. Realization of Try-Once-Discard in wireless multihop networks. IEEE Transactions on Industrial Informatics, 10(1):17-26, Feb 2014.

[6] D. Dimarogonas and K. Johansson. Event-triggered control for multi-agent systems. In Decision and Control, held jointly with the 28th Chinese Control Conference. CDC/CCC 2009. Proceedings of the 48th IEEE Conference on, pages 7131-7136, Dec 2009.

[7] M. Donkers, W. Heemels, D. Bernardini, A. Bemporad, and V. Shneer. Stability analysis of stochastic networked control systems. Automatica, 48(5):917-925, 2012.

[8] M. Gerasimenko, V. Petrov, O. Galinina, S. Andreev, and Y. Koucheryavy. Impact of machine-type communications on energy and delay performance of random access channel in LTE-advanced. Trans. Emerging Telecommun. Techn., 24(4):366-377, 2013.

[9] M. Hasan, E. Hossain, and D. Niyato. Random access for machine-to-machine communication in LTE-advanced networks: issues and approaches. IEEE Commun. Mag., 51(6):86-93, June 2013.

[10] W. P. M. H. Heemels, J. H. Sandee, and P. P. J. Van Den Bosch. Analysis of event-driven controllers for linear systems. International Journal of Control, 81(4):571-590, 2008.

[11] D. Kim, W. Kim, and S. An. Adaptive random access preamble split in LTE. In Proc. Int. Wireless Commun. and Mobile Computing Conf. (IWCMC), pages 814-819, July 2013.

[12] K. S. Ko, M. J. Kim, K. Y. Bae, D. K. Sung, J. H. Kim, and J. Y. Ahn. A Novel Random Access for Fixed-Location Machine-to-Machine Communications in OFDMA Based Systems. IEEE Commun. Letters, 16(9):1428-1431, September 2012.

[13] F. Kozin. A survey of stability of stochastic systems. Automatica, 5(1):95-112, Jan. 1969.

[14] A. Laya, L. Alonso, and J. Alonso-Zarate. Is the Random Access Channel of LTE and LTE-A Suitable for M2M Communications? A Survey of Alternatives. IEEE Commun. Surveys \& Tut., 16(1):4-16, First Qu. 2014 .
[15] K.-D. Lee, S. Kim, and B. Yi. Throughput comparison of random access methods for M2M service over LTE networks. In GLOBECOM Workshops ( $G C$ Wkshps), 2011 IEEE, pages 373-377, Dec 2011.

[16] S.-Y. Lien, T.-H. Liau, C.-Y. Kao, and K.-C. Chen. Cooperative Access Class Barring for Machine-to-Machine Communications. IEEE Trans. Wireless Commun., 11(1):27-32, January 2012.

[17] G. Madueno, S. Stefanovic, and P. Popovski. Efficient LTE access with collision resolution for massive M2M communications. In Proc. IEEE Globecom Workshops, pages 1433-1438, Dec 2014.

[18] M. Mamduhi, A. Molin, and S. Hirche. Event-based scheduling of multi-loop stochastic systems over shared communication channels. In 21st International Symposium on Mathematical Theory of Networks and Systems (MTNS), pages 266-273, Jul 2014.

[19] A. Molin and S. Hirche. On the optimality of certainty equivalence for event-triggered control systems. Automatic Control, IEEE Transactions on, 58(2):470 $-474,2013$.

[20] A. Molin and S. Hirche. A bi-level approach for the design of event-triggered control systems over a shared network. Discrete Event Dynamic Systems, 24(2):153-171, 2014.

[21] A. Molin and S. Hirche. Price-based adaptive scheduling in multi-loop control systems with resource constraints. Automatic Control, IEEE Transactions, pages 3282 - 3295, 2014.

[22] R. Murray, K. Astrom, S. Boyd, R. Brockett, and G. Stein. Future directions in control in an information-rich world. Control Systems, IEEE, 23(2):20-33, Apr 2003.

[23] D. Nesic and A. Teel. Input-output stability properties of networked control systems. Automatic Control, IEEE Transactions on, 49(10):1650-1667, 2004.

[24] C. Ramesh, H. Sandberg, and K. Johansson. Stability analysis of multiple state-based schedulers with CSMA. In Decision and Control (CDC), 2012 IEEE 51st Annual Conference on, pages 7205-7211, 2012.

[25] R. Rom and M. Sidi. Multiple Access Protocols: Performance and Analysis. Springer-Verlag New York, Inc., New York, NY, USA, 1990.

[26] M. Tabbara and D. Nesic. Input-output stability of networked control systems with stochastic protocols and channels. Automatic Control, IEEE Transactions on, 53(5):1160-1175, 2008.

[27] P. Tabuada. Event-triggered real-time scheduling of stabilizing control tasks. Automatic Control, IEEE Transactions on, 52(9):1680 -1685, 2007.

[28] D. Tolić and R. Fierro. Decentralized output synchronization of heterogeneous linear systems with fixed and switching topology via self-triggered communication. In American Control Conference, pages 4655-4660, June 2013.

[29] R. Tyagi, F. Aurzada, K.-D. Lee, S. Kim, and M. Reisslein. Impact of Retransmission Limit on Preamble Contention in LTE-Advanced Network. Systems Journal, IEEE, 9(3):752-765, Sept 2015.

[30] G. C. Walsh, H. Ye, and L. G. Bushnell. Stability analysis of networked control systems. Control 
Systems Technology, IEEE Transactions on, 10(3):438-446, 2002.

[31] G. Wu, S. Talwar, K. Johnsson, N. Himayat, and K. Johnson. M2M: From mobile to embedded Internet. IEEE Commun. Mag., 49(4):36-43, April 2011.

\section{APPENDIX}

\section{A. PROOF OF THEOREM 1}

Proof (Theorem 1). : To show LSP, let the NCS of interest operate over the interval $[k, k+N]$ of length $N$, starting from time-step $k$ with arbitrary initial state $e_{k}$. We assume that the NCS freely operates from the initial time $k$ until $k+N-1$ and we predict the error evolution considering all the possible scenarios under the introduced threshold-based scheduling policy over the interval $[k, k+N-1]$. Then, looking at the final time-step $k+N$, we show the aggregate error state $e_{k+N}$ fulfills (15). Depending on whether the condition (6) is satisfied at every time-step $k^{\prime}$, we divide the sub-systems $i \in\{1, \ldots, N\}$ into two complementary and disjoint sets as follows:

$$
i \in \begin{cases}\mathcal{G}_{k^{\prime}} & \left\|e_{k^{\prime}}^{i}\right\|>\Lambda_{i} \\ \overline{\mathcal{G}}_{k^{\prime}} & \left\|e_{k^{\prime}}^{i}\right\| \leq \Lambda_{i},\end{cases}
$$

where $\mathcal{G}_{k^{\prime}} \cup \overline{\mathcal{G}}_{k^{\prime}}=N$. According to (6), sub-systems belonging to $\mathcal{G}_{k^{\prime}}$ are eligible for transmission at time-step $k^{\prime}+1$. In accordance with the slotted ALOHA policy, if a sub-system $i$ is eligible for transmission at some time $k^{\prime}+1$, i.e. $\left\|e_{k^{\prime}}^{i}\right\|>\Lambda_{i}$, then $i$ selects one of the available transmission channels in uniform random fashion. If no other transmission-eligible sub-system $j \neq i$ selects that certain transmission channel, then sub-system $i$ successfully transmits. Otherwise, a collision occurs and both collided packets are dropped and the corresponding sub-systems will have to wait until the next time-step, i.e. $k^{\prime}+2$, to transmit, only if the inequality (6) is satisfied. To take this into account, we discern four complementary and mutually exclusive cases, covering the entire state space the error state $e_{k}$ evolves until time-step $k+N-1$ as follows:

Sub-system $i$ :

$c_{1}$ : has either successfully transmitted or not within the past $N-1$ time-steps, and is in set $\overline{\mathcal{G}}_{k+N-1}$, i.e.

$$
i \in \overline{\mathcal{G}}_{k+N-1} \quad \Rightarrow \quad\left\|e_{k+N-1}^{i}\right\| \leq \Lambda_{i},
$$

$c_{2}$ : has successfully transmitted at least once within the past $N-1$ time-steps, and is in set $\mathcal{G}_{k+N-1}$, i.e.

$$
\exists k^{\prime} \in[k, k+N-1]: \theta_{k^{\prime}}^{i}=1 \text { and }\left\|e_{k+N-1}^{i}\right\|>\Lambda_{i},
$$

$c_{3}$ : has not successfully transmitted within the past $N-1$ time-steps, and is in the set $\mathcal{G}_{k+N-1}$, but has been in the set $\overline{\mathcal{G}}_{k^{\prime}}$ at least once at some time-step $k^{\prime} \in[k, k+$ $N-2]$, i.e.

$$
\forall k^{\prime} \in[k, k+N-1]: \theta_{k^{\prime}}^{i}=0 \text { and }\left\|e_{k^{\prime}}^{i}\right\| \leq \Lambda_{i} .
$$

$c_{4}$ : has not successfully transmitted within the past $N-1$ time-steps, and has always been in the set $\mathcal{G}_{k^{\prime}}$ for all time-steps $k^{\prime} \in[k, k+N-1]$, i.e.

$$
\forall k^{\prime} \in[k, k+N-1]: \theta_{k^{\prime}}^{i}=0 \text { and }\left\|e_{k^{\prime}}^{i}\right\|>\Lambda_{i} .
$$

Introducing the above cases, we study the boundedness of error norm expectation over the interval $[k, k+N]$ for cases $c_{1}-c_{4}$. Since, the cases are complementary and mutually exclusive, i.e. each sub-system belongs exactly to one of the cases $c_{1}-c_{4}$, we can express (15) as

$$
\sum_{i=1}^{N} \mathrm{E}\left[\left\|e_{k+N}^{i}\right\|^{2}\right]=\sum_{i \in c_{l}}^{l=1,2,3,4} \mathrm{E}\left[\left\|e_{k+N}^{i}\right\|^{2} \mid c_{l}\right] .
$$

Suppose that some sub-systems $i$ belong to $c_{1}$, i.e. those sub-systems have never attempted for transmission. Since $i \in \overline{\mathcal{G}}_{k+N-1}$, it follows from (24) that $\left\|e_{k+N-1}^{i}\right\| \leq \Lambda_{i}$. Thus, those sub-systems are not eligible for transmission at timestep $k+N$, i.e. $\theta_{k+N}^{i}=0$. Then, it follows from (5) and (25) that

$$
\begin{aligned}
\sum_{i \in c_{1}} \mathrm{E} & {\left[\left\|e_{k+N}^{i}\right\|^{2} \mid e_{k}\right]=\sum_{i \in c_{1}} \mathrm{E}\left[\left\|A_{i} e_{k+N-1}^{i}+w_{k+N-1}^{i}\right\|^{2} \mid e_{k}\right] } \\
& \leq \sum_{c_{1}}\left\|A_{i}\right\|_{2}^{2} \mathrm{E}\left[\left\|e_{k+N-1}^{i}\right\|^{2} \mid e_{k}\right]+\mathrm{E}\left[\left\|w_{k+N-1}^{i}\right\|^{2}\right] \\
& \leq \sum_{c_{1}} \Lambda_{i}^{2}\left\|A_{i}\right\|_{2}^{2}+\mathrm{E}\left[\left\|w_{k+N-1}^{i}\right\|^{2}\right]
\end{aligned}
$$

This fulfills the condition (16) with $\bar{\xi}^{\prime}>\sum_{c_{1}} \Lambda_{i}^{2}\left\|A_{i}\right\|_{2}^{2}+$ $\mathrm{E}\left[\left\|w_{k+N-1}^{i}\right\|^{2}\right]$, and $\bar{\xi}=\frac{\sum_{c_{1}} \mathrm{E}\left[\left\|e_{k+N}^{i}\right\|^{2} \mid e_{k}\right]}{\bar{\xi}^{\prime}}<1$.

For some $i \in c_{2}$, let a successful transmission occur at timestep $k+r_{i}$, where $r_{i} \in[1, N-1]$, i.e. $\theta_{k+r_{i}}^{i}=1$. We express $e_{k+N}^{i}$ as a function of the error at time $k+r_{i}-1$ as

$$
\begin{aligned}
e_{k+N}^{i} & =\prod_{j=r_{i}}^{N}\left(1-\theta_{k+j}^{i}\right) A_{i}^{N-r_{i}+1} e_{k+r_{i}-1}^{i} \\
& +\sum_{r=r_{i}}^{N}\left[\prod_{j=r+1}^{N}\left(1-\theta_{k+j}^{i}\right) A_{i}^{N-r} w_{k+r-1}^{i}\right],
\end{aligned}
$$

where we define $\prod_{N+1}^{N}\left(1-\theta_{k+j}^{i}\right):=1$. The first term of the above equality vanishes as $\theta_{k+r_{i}}^{i}=1$. By statistical independence of $w_{k+r-1}^{i}$ and $\theta_{k+j}^{i}$, it follows from (27)

$$
\begin{aligned}
& \sum_{i \in c_{2}} \mathrm{E}\left[\left\|e_{k+N}^{i}\right\|^{2} \mid e_{k}\right] \\
& =\sum_{c_{2}} \mathrm{E}\left[\left\|\sum_{r=r_{i}^{\prime}}^{N} \prod_{j=r+1}^{N}\left[1-\theta_{k+j}^{i}\right] A_{i}^{N-r} w_{k+r-1}^{i}\right\|^{2}\right] \\
& \leq \sum_{c_{2}} \sum_{r=r_{i}^{\prime}}^{N} \mathrm{E}\left[\left\|A_{i}^{N-r} w_{k+r-1}^{i}\right\|^{2}\right] .
\end{aligned}
$$

Hence, the condition (16) is satisfied considering $\bar{\xi}^{\prime}$ chosen to be larger than (28), and $\bar{\xi}=\frac{\sum_{c_{2}} \mathrm{E}\left[\left\|e_{k+N}^{i}\right\|^{2} \mid e_{k}\right]}{\xi^{\prime}}<1$. Note that we assume to have only two transmission channels per time slot in this proof, therefore if the number of sub-systems which are eligible for transmission is greater than two, and one sub-system belongs to $c_{2}$, then the rest of the transmission eligible sub-systems belong to either set $c_{3}$ or $c_{4}$. This means that, one successful transmission occurs through one of the two available channels, while the other sub-systems which attempt to access the channel will not successfully transmit as they simultaneously select the second channel and eventually their corresponding data packet are collided.

For the case $c_{3}$, assume that the $k+r_{i}$ is the last timestep for sub-systems $i \in c_{3}$ that $i \in \overline{\mathcal{G}}_{k+r_{i}}$, which in turn implies that $\left\|e_{k+r_{i}}^{i}\right\| \leq \Lambda_{i}$. Recall that the sub-systems $i \in c_{3}$ belong to $\mathcal{G}_{k+N-1}$. Knowing that $\theta_{k^{\prime}}^{i}=0$ for $i \in c_{3}$ for all $k^{\prime} \in[k, k+N-1]$, we reach

$$
\begin{aligned}
& \sum_{c_{3}} \mathrm{E}\left[\left\|e_{k+N}^{i}\right\|^{2} \mid e_{k}\right] \leq \\
& \sum_{c_{3}}\left[\Lambda_{i}^{2}\left\|A_{i}^{N-r_{i}}\right\|_{2}^{2}+\sum_{r=r_{i}}^{N-1} \mathrm{E}\left[\left\|A_{i}^{N-r-1} w_{k+r}^{i}\right\|^{2}\right]\right] .
\end{aligned}
$$


The condition (16) is met by choosing $\bar{\xi}^{\prime}$ larger than the uniform upper bound (29), and $\bar{\xi}=\frac{\sum_{c_{3}} E\left[\left\|e_{k+N}^{i}\right\|^{2} \mid e_{k}\right]}{\bar{\xi}^{\prime}}<1$.

The sub-systems $i \in c_{4}$ have always been candidates for channel access, i.e. $i \in \mathcal{G}_{[k, k+N-1]}$, but they have never transmitted, which means that every single attempt from those sub-systems ended up with a collision. Hence, $\left\|e_{k^{\prime}}^{i}\right\|>\Lambda_{j}$ for all $k^{\prime} \in[k, k+N-1]$ while $\theta_{k^{\prime}}^{i}=0$. To show LSP in this case, we consider the worst case scenario by assuming every subsystem $i \in\{1, \ldots, N\}$ belongs to the set $c_{4}$, which instead ensures having successive collisions over the entire interval $[k, k+N]$. Indeed, we assume that every attempts to access one of the two available channels results in collisions and consequently no successful transmission would happen over the entire period. Generally, the probability that such a scenario happens for $M$ available transmission channels can be calculated as follows:

$$
\mathrm{P}_{\text {fail }}=\prod_{i=k}^{k+N} \mathrm{P}_{\text {fail }}^{i}
$$

where $\mathbf{P}_{\text {fail }}^{k}$ is the probability that all sub-systems collide in a given slot $k$ with $M$ channels. In general, the number of sub-systems eligible for transmission in a given slot $k$ is $g_{k}=\left|\mathcal{G}_{k}\right|$. Thus, the probability $\mathrm{P}_{\text {fail }}^{k}$ can be derived as:

$$
\mathrm{P}_{\text {fail }}^{k}=\frac{M^{g_{k}}-m_{1 s}}{M^{g_{k}}},
$$

where $M^{g_{k}}$ is the total number of possible channel choices for all transmitting sub-systems for a given slot, and $m_{1 s}$ is the number of outcomes with at least one successful transmission.

The probability of one specific sub-system to succeed is given in (9). As it can be any of $g_{k}$ sub-systems, and they can be successful with any channel, total number of such outcomes is defined as:

$$
g_{k} M\left(\frac{M-1}{M}\right)^{g_{k}}
$$

Now, by Inclusion-Exclusion principle, the probabilities of two successful transmissions in the slot $k$ are counted in (32) twice. There are exactly 2 ! ways for two success matches, and they can occur in for any pair channels for any pair of sub-systems, resulting in

$$
2 !\left(\begin{array}{c}
M \\
2
\end{array}\right)\left(\begin{array}{c}
g_{k} \\
2
\end{array}\right)(M-2)^{g_{k}-2}
$$

possible outcomes. Following the Inclusion-Exclusion principle, we need to subtract the number of outcomes with three successes, and so forth.
Thus, at the end, we can derive $m_{1 s}$ as follows:

$$
m_{1 s}=\sum_{j=1}^{\min \left(g_{k}, M\right)}(-1)^{j+1} \cdot j !\left(\begin{array}{c}
M \\
j
\end{array}\right)\left(\begin{array}{c}
g_{k} \\
j
\end{array}\right)(M-j)^{g_{k}-j}
$$

Using expression (34) in (31), we get the probability of all transmissions fail in one slot:

$$
\mathrm{P}_{\text {fail }}^{k}=\frac{M^{g_{k}}+\sum_{j=1}^{\min \left(g_{k}, M\right)}(-1)^{j} \cdot j !\left(\begin{array}{c}
M \\
j
\end{array}\right)\left(\begin{array}{c}
g_{k} \\
j
\end{array}\right)(M-j)^{g_{k}-j}}{M^{g_{k}}},
$$

Note that for any given slot, maximum number of eligible for transmission sub-systems is at most $N$, thus, $g_{k} \leq N$. Therefore, we can derive the upper bound on the $P_{\text {fail }}$ as:

$$
\mathrm{P}_{\text {fail }} \leq\left(\mathrm{P}_{\text {fail }}^{k}\right)^{N}
$$

with:

$$
\mathrm{P}_{\text {fail }}^{k} \leq \frac{M^{N}+\sum_{j=1}^{\min (N, M)}(-1)^{j} \cdot j !\left(\begin{array}{c}
M \\
j
\end{array}\right)\left(\begin{array}{c}
N \\
j
\end{array}\right)(M-j)^{N-j}}{M^{N}} .
$$

From (27), if no sub-system transmits over the $N$-step horizon, we can choose $\bar{\xi}^{\prime}=\sum_{i=1}^{N}\left\|A_{i}^{N} e_{k}^{i}+\sum_{r=1}^{N} A_{i}^{N-r} w_{k+r-1}^{i}\right\|^{2}>$ 0 . Therefore, we have

$$
\sup _{e_{k}} \mathrm{P}\left[\sum_{i=1}^{N}\left\|e_{k+N}^{i}\right\|^{2} \geq \bar{\xi}^{\prime}\right]<\mathrm{P}_{\text {fail }},
$$

for an arbitrary $\rho\left(\bar{\xi}^{\prime}, \bar{\xi}\right)$ such that $\sum_{i=1}^{N}\left\|e_{k}^{i}\right\|^{2}<\rho$ and LSP of the overall NCS is readily obtained according to (16).

REMARK 2. It should be noted that $\mathrm{P}_{\text {fail }}$ depends on the system parameters $\Lambda_{i}$ and $A_{i}$ and the noise covariance $W_{i}$ via the variable $g_{k}$. In fact, inclusion or exclusion of a subsystem $i$ in the set $\mathcal{G}_{k}$ does not only depend on the transmission history, but also on the system parameters and noise variables. 